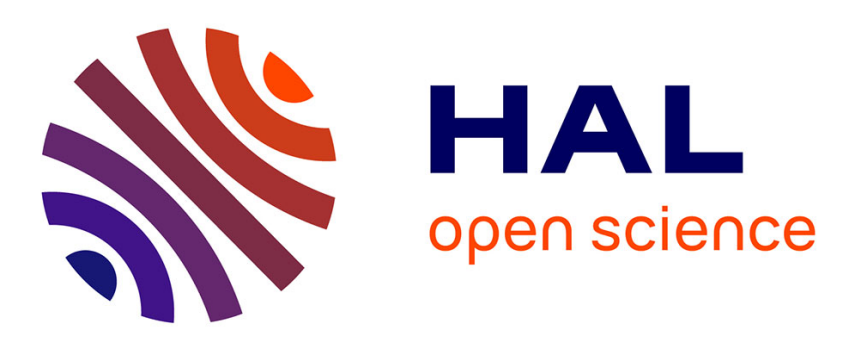

\title{
Comparing System Reliabilities with Ill-Known Probabilities
}

Lanting Yu, Sébastien Destercke, Mohamed Sallak, Walter Schon

\section{To cite this version:}

Lanting Yu, Sébastien Destercke, Mohamed Sallak, Walter Schon. Comparing System Reliabilities with Ill-Known Probabilities. 16th International Conference on Information Processing and Management of Uncertainty in Knowledge-Based Systems (IPMU 2016), Jun 2016, Eindhoven, Netherlands. pp.619-629, 10.1007/978-3-319-40581-0_50 . hal-01346452

\section{HAL Id: hal-01346452 \\ https://hal.science/hal-01346452}

Submitted on 19 Jul 2016

HAL is a multi-disciplinary open access archive for the deposit and dissemination of scientific research documents, whether they are published or not. The documents may come from teaching and research institutions in France or abroad, or from public or private research centers.
L'archive ouverte pluridisciplinaire HAL, est destinée au dépôt et à la diffusion de documents scientifiques de niveau recherche, publiés ou non, émanant des établissements d'enseignement et de recherche français ou étrangers, des laboratoires publics ou privés. 


\title{
Comparing system reliabilities with ill-known probabilities
}

\author{
Lanting Yu, Sébastien Destercke, Mohamed Sallak, and Walter Schon \\ Sorbonnes université, Université de Technologie de Compiègne, HEUDIASYC, UMR 7253. 57 \\ Avenue de Landshut. 60280 Compiegne, Francesurname name@hds .utc.fr
}

\begin{abstract}
In reliability analysis, comparing system reliability is an essential task when designing safe systems. When the failure probabilities of the system components (assumed to be independent) are precisely known, this task is relatively simple to achieve, as system reliabilities are precise numbers. When failure probabilities are ill-known (known to lie in an interval) and we want to have guaranteed comparisons (i.e., declare a system more reliable than another when it is for any possible probability value), there are different ways to compare system reliabilities. We explore the computational problems posed by such extensions, providing first insights about their pros and cons.
\end{abstract}

Keywords: System design, Reliability analysis, Imprecise probability, Comparison

\section{Introduction}

Being able to compare system reliabilities is essential when designing systems. Provided the structure function mapping single component reliabilities to the overall system reliability is known, this step poses no particular problem (at least from a theoretical standpoint) when failure probabilities are precisely known.

However, in practice, it may be difficult to provide precise assessments of such probabilities, for example because little data exist for the components (they may be issued from new technologies), or because they are given by expert opinions. This typically happens in early-stage phase design of new systems. In such a case, the problem of comparing system reliabilities become much more difficult, both conceptually and computationally speaking.

In this paper, we explore what happens when the component probabilities of functioning are ill-known, that is are only known to lie in an interval. Several aspects of reliability analysis have been extended to the case of ill-known probabilities, such as importance indices [8], multi-state systems [4], common cause failure problems [9], ... Yet, to our knowledge the problem of system reliability comparison remain to be formally studied within this setting.

In Section 3, we extend usual system comparisons (recalled in Section 2) to intervalvalued probabilities in two different ways, discussing the theoretical and practical pros and cons of each extension. Section 4 provides a more complex examples than the very simple, illustrative ones provided along the paper. The necessary basics of reliability as well as notations are briefly recalled in Section 2 


\section{System modelling and comparison: basics}

In this paper, we assume that we want to compare the designs of $K$ systems $S_{1}, \ldots, S_{K}$ in terms of reliability, in order to choose (one of) the safest among them. The $k$ th system will be composed of a set of $r^{k}$ components, and a given component can belong to one of $T$ populations (types) of components, all components of a population being assumed to have the same stochastic behaviour (i.e., same failure rate).

We will denote by $p_{j} \in[0,1]$ the possibly ill-known probability that a component of type $j$ is functioning, and $1-p_{j}$ the probability that it is inoperative or malfunctioning. We will also denote by $x_{i, j_{i k}}^{k} \in\{0,1\}$ both the $i$ th component of $k$ th system, which is of type $j_{i k}$, as well as its state ( $x_{i, j_{i k}}^{k}=0$ if malfunctioning, 1 if working). $p_{j_{i k}}$ is then the probability of $x_{i, j_{i k}}^{k}=1$. Table 1 summarises these notations.

\begin{tabular}{ccc}
\hline Variable & Domain & Meaning \\
\hline$K$ & $\mathbb{Z}$ & Number of systems \\
$r^{k}$ & $\mathbb{Z}$ & Number of components in the $k$ th system \\
$T$ & $\mathbb{Z}$ & Number of component types (of possible stochastic behaviors) \\
$p_{j}$ & {$[0,1], j \in\{1, \ldots, T\}$} & Probability that a component of type $j$ will be working \\
$x_{i, j_{i k}}^{k}$ & $\{0,1\}$ & $i$ th component of $k$ th system, of type $j_{i k}$, and its state \\
\hline & & Table 1. Notation summary
\end{tabular}

In this paper, we will assume that we know the structure function $\phi^{k}:\{0,1\}^{r^{k}} \rightarrow$ $\{0,1\}$ of the $k$ th system and that it is written in the "simple" following way:

$$
\phi^{k}\left(x_{1, j_{1 k}}^{k}, \ldots, x_{r_{k}, j_{r_{k k}}}^{k}\right)=\sum_{A \subseteq\left\{1, \ldots, r_{k}\right\}} d_{A}^{k} \prod_{i \in A} x_{i, j_{i k}}^{k}
$$

with $d_{A}^{k}$ real-valued coefficients (some subsets $A$ can receive $d_{A}=0$ ) that can either be positive or negative. At least in principle, every system and structure function can be put in the form of Eq. (1), that is a multi-linear form [2]. We also make the classical assumption in reliability that each system is coherent, meaning that $\phi^{k}$ is increasing ${ }^{1}$ and that we have the boundary condition $s^{2} \phi^{k}(0, \ldots, 0)=0, \phi^{k}(1, \ldots, 1)=1$. Going from the structure function to the reliability $R^{k}$ of the system is then quite simple, as it simply consists in replacing $x_{i, j_{i k}}$ by the corresponding probability $p_{j_{i k}}$, that is

$$
R^{k}\left(p_{j_{1 k}}, \ldots, p_{j_{r_{k} k}}\right)=\sum_{A \subseteq\left\{1, \ldots, r_{k}\right\}} d_{A}^{k} \prod_{i \in A} p_{j_{i k}}
$$

To simplify notations, we will simply note $R^{k}\left(p_{j_{1 k}}, \ldots, p_{j_{r_{k}}}\right)$ by $R^{k}$. Note that $R^{k}$ is a function of the probabilities $p_{j}$, that can appear multiple times for one subset $A$. Note that being a coherent system means that the functions $R^{j}$ are increasing in every variable $p_{j}$.

\footnotetext{
${ }^{1}$ If one component goes from failing to working, then the system state can only improve.

2 The system works (fails) if all components work (fail).
} 
Example 1. Assume we have two (very) simple series system $(K=2)$, a first with two components, a second with three, and three different component types $(T=3)$. The two first components of each system are of the same type ( 1 and 2, respectively). The systems are illustrated in Figure 1, and we do have

$$
\begin{gathered}
R^{1}=p_{1} \cdot p_{2} \\
R^{2}=p_{1} \cdot p_{2} \cdot p_{3}
\end{gathered}
$$

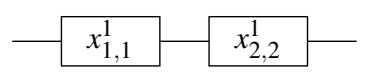

System 1

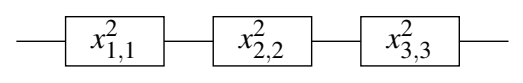

System 2

Fig. 1. Two simple series systems

Comparing two systems (say, the $k$ th and $\ell$ th) then comes down to compare their reliabilities $R^{k}$ and $R^{\ell}$. System $S_{k}$ is then said to be preferred to system $S_{\ell}$, denoted by $S_{k} \succ S_{\ell}$, if and only if

$$
R^{k}>R^{\ell}
$$

or, equivalently when probabilities $p_{j}$ are precisely known, if and only if

$$
R^{k}-R^{\ell}>0 .
$$

Example 2. Let us continue Example 1 by using the precisely valued probabilities $p_{1}=$ $0.8, p_{2}=0.9$ and $p_{3}=0.8$. We then have

$$
R^{1}=0.72 \text { and } R^{2}=0.576
$$

meaning that system $S_{2}$ should be discarded. We also have

$$
R^{1}-R^{2}=p_{1} \cdot p_{2}-p_{1} \cdot p_{2} \cdot p_{3}=p_{1} \cdot p_{2} \cdot\left(1-p_{3}\right)=0.144 .
$$

We can also notice that whatever the values of $p_{1}, p_{2}, p_{3}$, we will always have $S_{1} \succ S_{2}$ (since $R^{1}-R^{2}$ is a product of positive terms).

Let us now investigate what becomes of such a comparison when probabilities $p_{j} \in$ $\left[\underline{p}_{j}, \bar{p}_{j}\right]$ are only known to lie in intervals.

\section{Comparing systems with interval probabilities}

In this section, we investigate the most natural extensions of Equations (3) and (4) to an imprecise setting. We will see that in the imprecise case, they do no longer coincide, and the first extension only provides an approximation of the second one, but is computationally more tractable. 
Note that in this paper, we are interested in guaranteed comparisons, that is we want to assess that $S^{k}$ is more reliable than $S^{\ell}$ when this is true for any values of $p_{j}$ within $\left[\underline{p}_{j}, \bar{p}_{j}\right]$ and for $j=1, \ldots, T$. For convenience, we will denote by $\mathscr{P}=\times_{j=1}^{T}\left[\underline{p}_{j}, \bar{p}_{j}\right]$ the Cartesian product of those intervals.

\subsection{Interval comparison: definition}

A first way to extend the comparison is to compute bounds over $R^{k}$, obtaining the interval $\left[\underline{R}^{k}, \bar{R}^{k}\right]$ such that

$$
\underline{R}^{k}=\inf _{p_{j_{i k}} \in\left[\underline{p}_{j_{i k}}, \bar{p}_{j_{i k}}\right]} R^{k}=\sum_{A \subseteq\left\{1, \ldots, r_{k}\right\}} d_{A}^{k} \prod_{i \in A} \underline{p}_{j_{i k}}
$$

and

$$
\bar{R}^{k}=\inf _{p_{j_{i k}} \in\left[\underline{p}_{j_{i k}}, \bar{p}_{j_{k}}\right]} R^{k}=\sum_{A \subseteq\left\{1, \ldots, r_{k}\right\}} d_{A}^{k} \prod_{i \in A} \bar{p}_{j_{i k}} .
$$

Where the fact that probability values can be replaced by their corresponding bounds follows from the increasing monotonicity of reliability functions. We can then straightforwardly extend Eq. (3) by saying that system $S^{k}$ is interval-preferred to system $S^{\ell}$, denoted $S^{k} \succ_{I C} S^{\ell}$, if and only if

$$
\underline{R}^{k}>\bar{R}^{\ell}
$$

that is we are absolutely certain that $S^{k}$ is more reliable than $S^{\ell}$. In this case, comparing two systems just come down to make four computations instead of two to get the corresponding intervals. If the two intervals overlap, then systems $S^{k}$ and $S^{\ell}$ are incomparable according to this criterion.

However, comparison (7) is very rough, in the sense that it will often result in incomparability of systems, even if it is obvious that one system is preferable to another, as example 3 shows.

Example 3. Let us consider the systems of Example 2 with the following bounds

$$
p_{1} \in[0.7,0.9], p_{2} \in[0.8,1] \text { and } p_{3} \in[0.7,0.9] \text {. }
$$

We then obtain the intervals

$$
R^{1} \in[0.56,0.9] \text { and } R^{2} \in[0.392,0.81]
$$

meaning that the system are not comparable according to $\succ_{I C}$.

\subsection{Difference comparison:definition}

Interval comparison somehow extends Equation (3), but a second way to extend the precise comparison is to extend Equation (4). Before doing so, let us simplify notations 
by adopting the convention that $R^{k-\ell}:=R^{k}-R^{\ell}$. We can then say that system $S^{k}$ is difference-preferred to system $S^{\ell}$, denoted $S^{k} \succ_{D C} S^{\ell}$, if and only if the value

$$
\begin{aligned}
& \underline{R}^{k-\ell}=\inf _{\substack{p_{j_{i k}} \in\left[p_{j_{i k}}, \bar{p}_{j_{k}}\right] \\
p_{j_{i} \ell} \in\left[p_{j_{i}}, \bar{p}_{j_{i \ell}}\right]}} R^{k}-R^{\ell} \\
& =\inf _{\substack{p_{j_{i k}} \in\left[p_{j_{i k}}, \bar{p}_{j_{i k}}\right]_{A \subseteq\left\{1, \ldots, r_{k}\right\}} \\
p_{j_{i \ell} \ell} \in\left[p_{i,}, \bar{p}_{j_{i \ell}}\right]}} d_{A}^{k} \prod_{i \in A} p_{j_{i k}}-\sum_{A \subseteq\left\{1, \ldots, r_{\ell}\right\}} d_{A}^{\ell} \prod_{i \in A} p_{j_{i \ell}}
\end{aligned}
$$

is positive, i.e., $\underline{R}^{k-\ell}>0$. In practice, this comes down to ask $R^{k}$ to be higher than $R^{\ell}$ for all possible values of $p_{j}$, hence it also gives a guaranteed comparison. Example 4 and Corollary 1 show that this way of comparing systems is actually better than the previous, in the sense that it still gives guarantee but is less conservative. Yet, computing $\underline{R}^{k-\ell}$ can be far from straightforward (in contrast with the case of interval comparison), and we try to characterize in the next section when this task will be easy.

Example 4. Let us apply Equation (7) to Example 3 In this case we have from Example 2 that $R^{1-2}=p_{1} \cdot p_{2} \cdot\left(1-p_{3}\right)$ and so

$$
\underline{R}^{1-2}=\inf _{\substack{p_{1} \in[0.7,0.9], p_{2} \in[0.8,1], p_{3} \in[0.7,0.9]}} p_{1} \cdot p_{2} \cdot\left(1-p_{3}\right)=0.7 \cdot 0.8 \cdot 0.1=0.056
$$

which is indeed quite low, but still higher than zero, hence $S^{1} \succ_{D C} S^{2}$, allowing us to reach a decision where we could not before.

And indeed, we always have the following relation between the two notions:

Proposition 1. $\underline{R}^{k-\ell} \geq \underline{R}^{k}-\bar{R}^{\ell}$

Proof. The inequality $\inf _{x \in D} f(x)+g(x) \geq \inf _{x \in D} f(x)+\inf _{x \in D} g(x)$ with $x$ a vector of values and $D$ a convex set is known to be true. If we define $x$ as the vector of probability values $p_{1}, \ldots, p_{T}$, and take $f=R^{k}, g=-R^{\ell}, D=\mathscr{P}$, we get

$$
\inf _{x \in \mathscr{P}} R^{k}(x)-R^{\ell}(x) \geq \inf _{x \in \mathscr{P}} R^{k}(x)+\inf _{x \in \mathscr{P}}-R^{\ell}(x) \geq \inf _{x \in \mathscr{P}} R^{k}(x)-\sup _{x \in \mathscr{P}} R^{\ell}(x)
$$

We then get the following corollary, showing that if $S^{k} \succ_{I C} S^{\ell}$, then $S^{k} \succ_{D C} S^{\ell}$, but not the reverse. Actually, a similar problem is known under the name "dependency problem" in interval arithmetic, for which many solutions have been proposed [3].

Corollary 1. If $\underline{R}^{k}-\bar{R}^{\ell}>0$, then $\underline{R}^{k-\ell}>0$

So $\underline{R}^{k-\ell}$ is definitely a more accurate way of comparing systems. Let us now study a bit the problem of actually computing it. 
Remark 1. In imprecise probability theory, a similar relation exists between the maximality decision rule and the interval dominance decision rule [10]. However, two main differences, in terms of optimization problems, between imprecise probabilities and our study are that $\mathscr{P}$ is here an hypercube and that optimization has to be done over nonlinear functions in general, while imprecise probabilities is concerned with bounds of expectations over a subset of the unit simplex. Note that we could also search to adapt other imprecise probability decision rules: maximin and maximax extend directly by using Equations (5) and (6), while the notion of E-admissibility may require more involved investigation, especially as it is not based on a pairwise comparison scheme.

\subsection{Computing $\underline{R}^{k-\ell}$}

In general, $R^{k}-R^{\ell}$ will be a polynomial in variables $p_{j}$ that is neither decreasing nor increasing in those variables. Computing bounds over such polynomials when variables lie in a hyper-cube (which is our case) is known to be NP-hard [6], hence infeasible in practice. Two solutions are then to look for approximations that remain close to $\underline{R}^{k-\ell}$ but are more tractable (using interval bounds provides a crude approximation), or to identify those sub-cases for which the solution will be easier to find. In this paper, we explore the second alternative, and leave the first for future works.

Before studying in detail how $\underline{R}^{k-\ell}$ can be computed, we have to recall the notions of global monotonicity and of local monotonicity of a function [5] $f\left(x_{1}, \ldots, x_{n}\right)$ in a variable $x_{i}$

Definition 1 (Global monotonicity). Function $f\left(x_{1}, \ldots, x_{n}\right)$ is globally increasing (decreasing) in $x_{i}$ if it is always increasing (decreasing) in $x_{i}$, irrespectively of the other variable values.

If $f$ is globally increasing in $x_{i}$, then its lower and upper bounds are known to be obtained for $x_{i}=\underline{x}_{i}$ and $x_{i}=\bar{x}_{i}$ when $x_{i} \in\left[\underline{x}_{i}, \bar{x}_{i}\right]$, respectively.

Definition 2 (Local monotonicity). Function $f\left(x_{1}, \ldots, x_{n}\right)$ is locally increasing (decreasing) in $x_{i}$ if it is either increasing or decreasing in $x_{i}$ when the other variables $x_{1}, \ldots, x_{i-1}, x_{i+1}, \ldots, x_{n}$ values are fixed.

If $f$ is locally monotonic in $x_{i}$, then its bounds are known to be obtained for $x_{i}=$ $\underline{x}_{i}$ or $x_{i}=\bar{x}_{i}$, but which value to take between these two ones depends on the other variable values, in contrast with global monotonicity (where the value to consider is fixed, whatever the other variable values). A function will be said to be non-monotone in $x_{i}$ if it is not locally or globally monotone in it.

Example 5. Consider the following functions of $x_{1}, x_{2}$ with $x_{i} \in[-2,1]$, then the functions

$$
f_{1}\left(x_{1}, x_{2}\right)=x_{1}-x_{2}, \quad f_{2}\left(x_{1}, x_{2}\right)=-\left(x_{1} \cdot x_{2}\right), \quad f_{3}\left(x_{1}, x_{2}\right)=x_{1}^{2} \cdot x_{2}^{2}
$$

are respectively globally, locally, and not monotone in each of their variables. $x_{1}\left(x_{2}\right)$ is globally increasing (decreasing) in $f_{1} \cdot f_{2}$ is decreasing in $x_{1}\left(x_{2}\right)$ when $x_{2}\left(x_{1}\right)$ is positive, and increasing when $x_{2}\left(x_{1}\right)$ is negative (hence the monotonicity depends on the value of the other variables). $f_{3}$ is neither locally nor globally monotone in both variables (i.e., $\underline{f}_{3}=f_{3}(0,0)$ ). 
Given two systems $S^{k}$ and $S^{\ell}$, we now define the following subsets of component types:

- The subsets

$$
\begin{aligned}
& T_{k}=\left\{j \in\{1, \ldots, T\} \mid \forall p_{j_{i \ell}}, i=1, \ldots, r^{\ell}, j_{i \ell} \neq j\right\} \\
& T_{\ell}=\left\{j \in\{1, \ldots, T\} \mid \forall p_{j_{i k}}, i=1, \ldots, r^{k}, j_{i k} \neq j\right\}
\end{aligned}
$$

that denote the types of components that are encountered only in system $S^{k}\left(T_{k}\right)$ or $S^{\ell}\left(T_{\ell}\right)$.

- The subset

$$
\begin{gathered}
T_{\ell \cap k, 1}=\left\{j \in\{1, \ldots, T\} \mid \exists p_{j_{i \ell}, p_{j_{i^{\prime} k}} \text { s.t. } j_{i \ell}=j_{i^{\prime} k}=j}=\wedge\right. \\
\exists ! i \text { s.t. } j_{i \ell}=j \\
\left.\exists ! i^{\prime} \text { s.t. } j_{i^{\prime} k}=j\right\}
\end{gathered}
$$

that includes all component types that are in both systems, but only once in each of them.

- The subset

$$
\begin{aligned}
& T_{\ell \cap k,+}=\left\{j \in\{1, \ldots, T\} \mid \exists p_{j_{i \ell}}, p_{j_{i^{\prime} k}} \text { s.t. } j_{i \ell}=j_{i^{\prime} k}=j \wedge \wedge\right. \\
& \left(\exists i, i^{\prime} \text { s.t. } j_{i \ell}=j_{i^{\prime} \ell}=j\right. \\
& \left.\left.\exists i, i^{\prime} \text { s.t. } j_{i k}=j_{i^{\prime} k}=j\right)\right\}
\end{aligned}
$$

that includes all component types that are in both systems and appear more than once in at least one of the two systems.

The subsets $T_{k}, T_{\ell}, T_{\ell \cap k, 1}, T_{\ell \cap k,+}$ form a well-defined partition of the component types in systems $S^{k}$ and $S^{\ell}$. We can then show a first property

Proposition 2. $R^{k}-R^{\ell}$ is a globally monotonic function in variables $p_{j}, j \in T_{k} \cup T_{\ell}$. It is increasing (decreasing) in variables $p_{j}, j \in T_{k}\left(p_{j}, j \in T_{\ell}\right)$

Proof. Without loss of generality, let us assume that $p_{1}, \ldots, p_{i} \in T_{k}$ and $p_{i+1}, \ldots, p_{j} \in$ $T_{\ell}$. By assumption, we have

$$
R^{k}-R^{\ell}=R^{k}\left(p_{1}, \ldots, p_{i}, p_{j+1}, \ldots, p_{T}\right)-R^{\ell}\left(p_{i+1}, \ldots, p_{T}\right),
$$

therefore the monotonicity with respect to $p_{1}, \ldots, p_{i}\left(p_{i+1}, \ldots, p_{j}\right)$ depends only of their monotonicity with respect to $R^{k}\left(R^{\ell}\right)$, which are known to both be increasing in those variables.

This means that if $p_{i} \in T_{k}$ or $T_{\ell}$, we know for which value of $p_{i}$ the lower bound is obtained $\left(\underline{p}_{i}\right.$ if $p_{i} \in T_{k}$, else $\left.\bar{p}_{i}\right)$. Also note that in the particular case where $T_{k \cap \ell, 1}=$ $T_{k \cap \ell,+}=\emptyset$, the following result follows: 
Lemma 1. if $T_{k \cap \ell, 1}=T_{k \cap \ell,+}=\emptyset$, then $\underline{R}^{k}-\bar{R}^{\ell}=\underline{R}^{k-\ell}$

Proof. When $T_{k \cap \ell, 1}=T_{k \cap \ell,+}=\emptyset$, there are no shared variables between $R^{k}$ and $R^{\ell}$, meaning that $j=T$ in proof of Prop 2 and that

$$
\inf _{p_{1}, \ldots, p_{i} p_{i+1, \ldots, T}} \inf _{p^{\prime}} R^{k}-R^{\ell}=R^{k}\left(\underline{p}_{1}, \ldots, \underline{p}_{i}\right)-R^{\ell}\left(\bar{p}_{i+1}, \ldots, \underline{p}_{T}\right)
$$

Proposition 3. $R^{k}-R^{\ell}$ is a locally monotonic function in variables $p_{j}, j \in T_{k \cap \ell, 1}$.

Proof. (sketch) We know that both $R^{k}$ and $R^{\ell}$ are equivalent to replacing the $x_{i, j_{i k}}^{k}$ in Equation (1) by their probability types. If a type $p_{i}$ of component is present once (and exactly once) in each system, this means that for every subset $A, p_{j}$ power will be either zero or one in the products $\prod_{i \in A^{k}} p_{j_{i k}}$ and $\prod_{i \in A^{\ell}} p_{j_{i \ell}}$ of Equation (2). Therefore, $R^{k}-R^{\ell}$ will be a sum of products where $p_{j}$ has power zero or one, meaning that if the other variables $p_{1}, \ldots, p_{j-1}, p_{j+1}, \ldots, p_{T}$ are fixed, the derivative of $R^{k}-R^{\ell}$ with respect to $p_{j}$ will be a constant (whose positivity or negativity will depend of $p_{1}, \ldots, p_{j-1}, p_{j+1}, \ldots, p_{T}$ values), hence that $R^{k}-R^{\ell}$ is either decreasing or increasing in $p_{j}$.

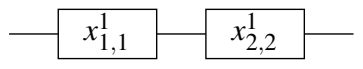

System 1

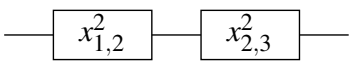

System 2

Fig. 2. Two simple series systems with common component

Example 6. Let us consider the series systems of Figure 2 with three types of components, where $p_{2} \in T_{1 \cap 2,1}$. We have

$$
R^{1}-R^{2}=p_{1} \cdot p_{2}-p_{3} \cdot p_{2}=p_{2} \cdot\left(p_{1}-p_{3}\right)
$$

which is indeed locally, but not globally, monotone in $p_{2}$ (it is increasing if $p_{1}>p_{3}$, decreasing if $p_{1}<p_{3}$ ).

This means that, if we have $N=\left|T_{k \cap \ell, 1}\right|$ variables $p_{j}$ for which we are locally monotone, we know that the lower bound is obtained for one of the $2^{N}$ vertices of the corresponding hypercube $\times_{i \in T_{k \cap \ell, 1}}\left[\underline{p}_{i}, \bar{p}_{i}\right]$. If $N$ is not too high, then we can think of simply enumerating the set of possible values.

Finally, we cannot guarantee any kind of monotonicity for the variables $p_{j}, j \in$ $T_{\ell \cap k,+}$. However, if the cardinality of $T_{\ell \cap k,+}$ is not too high, it is always possible to make a random search within its defined area. 
Example 7. Let us consider the very simple case depicted in Figure 3, where we have

$$
R^{1}=p_{1}^{2} \text { and } R^{2}=p_{1}
$$

hence $R^{1-2}=p_{1}^{2}-p_{1}=p_{1}\left(p_{1}-1\right)$, which will always be negative. However, if $p_{1} \in$ $[0.4,0.6]$, the bound $\underline{R}^{1-2}=-0.25$ is obtained for $p_{1}=0.5$, which does not correspond to one of the bounds $\underline{p}_{1}, \bar{p}_{1}$.

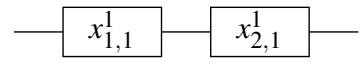

System 1

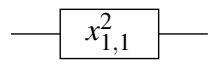

System 2

Fig. 3. Two simple series systems with redundancy

It means that when confronted with too much components present in both systems and multiple times in at least one of them, computing the bound may quickly become intractable in practice. This becomes even truer if the monotonicity of other variables (those in $T_{\ell \cap k, 1}$ ) depends on those variables in $T_{\ell \cap k,+}$.

An easy solution is to "duplicate" each variable $p_{j}$ in $T_{\ell \cap k,+}$ with variables having the same interval bound, so that each variable is present at most once in each system. In the case of Figure 3 , this means considering a variable $p_{1}^{\prime}$ for the second component of System 1. Such a straightforward approach has two potential drawbacks: the increase of the number of component types in $T_{\ell \cap k, 1}$, and the fact that the approximation can be quite loose. Such a strategy is therefore likely to be useful only when the number of component types.

\section{A more complex example}

Let us now consider two slightly more complex systems, where we want to chose the most reliable design. The systems are depicted by Figure 4 and consider three types of components, with $p_{1} \in[0.9,1], p_{2} \in[0.8,0.9]$ and $p_{3} \in[0.85,0.95]$, where one hesitates between choosing a 2 out of 3 architecture with slightly less reliable components, and a parallel architecture with potentially more reliable components. The reliabilities of the systems are

$$
R^{1}=p_{1} \cdot p_{2}^{2} \cdot\left(3-2 p_{2}\right)
$$

and

$$
R^{2}=p_{1} \cdot p_{3} \cdot\left(2-p_{3}\right)
$$

Intervals $\left[\underline{R}^{1}, \bar{R}^{1}\right]$ and $\left[\underline{R}^{2}, \bar{R}^{2}\right]$ intersect, hence interval comparison is not sufficient to tell us whether $S_{1}$ is better than $S_{2}$, or the reverse. We have $T_{1 \cap 2,1}=\{1\}, T_{1}=\{2\}$ and $T_{2}=\{3\}$, therefore if we want to compute $\underline{R}^{1-2}$, our previous results tell us that

$$
\underline{R}^{1-2}=p_{1}^{*} \cdot \underline{p}_{2}^{2} \cdot\left(3-2 \underline{p}_{2}\right)-p_{1}^{*} \cdot \bar{p}_{3} \cdot\left(2-\bar{p}_{3}\right)
$$


with $p_{1}^{*} \in\left\{p_{1}, \bar{p}_{1}\right\}$. The result is obtained for $\bar{p}_{1}$ (the function is decreasing in $p_{1}$ for $p_{2}=p_{2}$ and $p_{3}=\bar{p}_{3}$ ), and $\underline{R}^{1-2}=-0.1015$, meaning that we cannot conclude that $S_{1} \succ_{D C} S_{2}$. Following a similar line of reasoning for $\underline{R}^{2-1}$ (which is increasing in $p_{1}$ ), we get $\underline{R}^{2-1}=0.00495$ and are able to tell that $S_{2} \succ_{D C} S_{1}$.

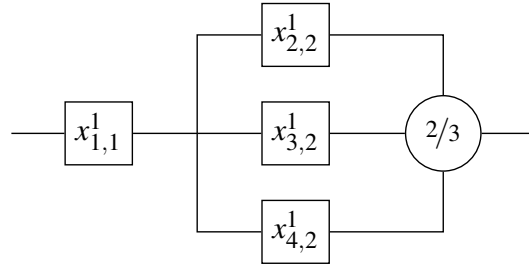

Fig.4A: System $S_{1}$

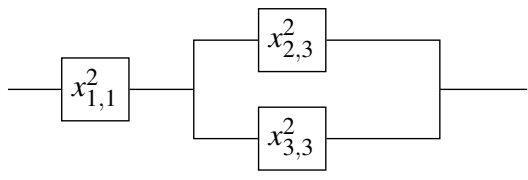

Fig.4B: System $S_{2}$

Fig. 4. Two system designs to compare.

\section{Conclusion}

In this paper, we have studied how comparisons of system reliabilities can be extended when probabilities are ill-known, or interval-valued. In particular, we have focused on comparison notions that allows for incomparability when the information is too weak to be certain that one system is more reliable than another.

We have seen that computing the lower bound over the difference of reliabilities is less conservative, but more computationally demanding than just comparing reliability bounds of each systems taken individually. While we have pointed out ways to reduce the complexity of such computations (by focusing on global and local comonotonicity), it remains to investigate how to approximate $\underline{R}^{k-\ell}$ with a lower bound better than $\underline{R}^{k}-$ $\bar{R}^{\ell}$, but computationally more tractable than computing $\underline{R}^{k-\ell}$. A first way to do so is to exploit bounds used when the reliability probabilities are precisely known, but when computing the output probability is computationally prohibitive, see e.g., [7].

An additional interesting problem to explore is to formalize which information we should query to make two incomparable systems comparable. For instance, we may formulate it as an expert elicitation problem [1].

\section{References}

1. Abdallah, N.B., Destercke, S.: Optimal expert elicitation to reduce interval uncertainty. In: Proceedings of the Thirty-First Conference on Uncertainty in Artificial Intelligence, UAI 2015, July 12-16, 2015, Amsterdam, The Netherlands. pp. 12-21 (2015)

2. Borgonovo, E.: The reliability importance of components and prime implicants in coherent and non-coherent systems including total-order interactions. European Journal of Operational Research 204(3), 485-495 (2010) 
3. De Figueiredo, L.H., Stolfi, J.: Affine arithmetic: concepts and applications. Numerical Algorithms 37(1-4), 147-158 (2004)

4. Ding, Y., Lisnianski, A.: Fuzzy universal generating functions for multi-state system reliability assessment. Fuzzy Sets and Systems 159(3), 307-324 (2008)

5. Fortin, J., Dubois, D., Fargier, H.: Gradual numbers and their application to fuzzy interval analysis. IEEE Transactions on Fuzzy Systems 16(2), 388-402 (2008)

6. Kreinovich, V., Lakeyev, A., Rohn, J.: Computational complexity of interval algebraic problems: Some are feasible and some are computationally intractable-a survey. Mathematical Research 90, 293-306 (1996)

7. Mteza, P.Y.: Bounds for the reliability of binary coherent systems. Ph.D. thesis (2014)

8. Sallak, M., Schon, W., Aguirre, F.: Extended component importance measures considering aleatory and epistemic uncertainties. Reliability, IEEE Transactions on 62(1), 49-65 (2013)

9. Troffaes, M.C., Walter, G., Kelly, D.: A robust bayesian approach to modeling epistemic uncertainty in common-cause failure models. Reliability Engineering \& System Safety 125, 13-21 (2014)

10. Troffaes, M.: Decision making under uncertainty using imprecise probabilities. Int. J. of Approximate Reasoning 45, 17-29 (2007) 